\title{
Derived Equivalence Classification of the Gentle Two-Cycle Algebras
}

\author{
Grzegorz Bobiński ${ }^{1}$
}

Received: 29 September 2015 / Accepted: 26 December 2016 / Published online: 9 January 2017

(C) The Author(s) 2017. This article is published with open access at Springerlink.com

\begin{abstract}
We complete a derived equivalence classification of the gentle two-cycle algebras initiated in earlier papers by Avella-Alaminos and Bobiński-Malicki.
\end{abstract}

Keywords Gentle two-cycle algebra $\cdot$ Derived category $\cdot$ Derived equivalence

Mathematics Subject Classification (2010) $16 \mathrm{G} 20 \cdot 18 \mathrm{E} 30$

\section{Introduction and the Main Result}

Throughout the paper $k$ denotes a fixed algebraically closed field. For a (finite-dimensional basic connected) algebra $\Lambda$ one considers its (bounded) derived category $\mathcal{D}^{b}(\Lambda)$, which has a structure of a triangulated category. Derived categories seem to be a proper setup to do homological algebra. Derived categories appearing in representation theory of algebras have connections with derived categories studied in algebraic geometry (see for example [11, 24, 31]). Moreover, these categories serve as a source for constructions of categorifications of cluster algebras (this line of research was initiated by a fundamental paper by Buan, Marsh, Reineke, Reiten and Todorov [20]) and have links to theoretical physics (including famous Orlov's theorem [36]).

Algebras $\Lambda^{\prime}$ and $\Lambda^{\prime \prime}$ are said to be derived equivalent if the categories $\mathcal{D}^{b}\left(\Lambda^{\prime}\right)$ and $\mathcal{D}^{b}\left(\Lambda^{\prime \prime}\right)$ are triangle equivalent. A study of derived categories (in particular derived equivalences) in the representation theory of algebras was initiated by papers of Happel [28,

Presented by Henning Krause.

Grzegorz Bobiński

gregbob@mat.umk.pl

1 Faculty of Mathematics and Computer Science, Nicolaus Copernicus University, ul. Chopina 12/18, 87-100 Toruń, Poland 
29] and motivated by tilting theory, and is now an important direction of research (see for example $[3,10,13,15,16,18,19,21,25,30,32,34,35,37,38])$.

Gentle algebras were introduced by Assem and Skowroński [6] in their study of the algebras derived equivalent to the hereditary algebras of Euclidean type $\tilde{\mathbb{A}}$. Namely, they have proved that the algebras derived equivalent to the hereditary algebras of Euclidean type $\tilde{\mathbb{A}}$ are precisely the gentle one-cycle algebras which satisfy the clock condition. On the other hand, the algebras derived equivalent to the hereditary algebras of Dynkin type $\mathbb{A}$ are precisely the gentle tree algebras [4]. Moreover, the gentle one-cycle algebras which do not satisfy the clock condition are precisely the discrete derived algebras, which are not locally finite [42]. The above motivates study of a derived equivalence classification for the gentle algebras. One should note that the class of gentle algebras is closed with respect to the derived equivalence [40].

By the above results the derived equivalence classes of the gentle algebras with at most one-cycle are known and they are distinguished by the invariant of Avella-Alaminos and Geiss [8]. It is natural to study as the next step a derived equivalence classification of the gentle two-cycle algebras. Here a gentle algebra $\Lambda$ is called two-cycle if the number of edges in the Gabriel quiver of $\Lambda$ exceeds by one the number of vertices in this quiver. Before formulating the main result of the paper we define some families of gentle two-cycle algebras.

By $\mathbb{Z}, \mathbb{N}$ and $\mathbb{N}_{+}$we denote the sets of integers, nonnegative integers and positive integers, respectively. If $i$ and $j$ are integers, then $[i, j]$ denotes the set of integers $l$ such that $i \leq l \leq$ $j$. For $p \in \mathbb{N}_{+}$and $r \in[0, p-1], \Lambda_{0}(p, r)$ is the algebra of the quiver

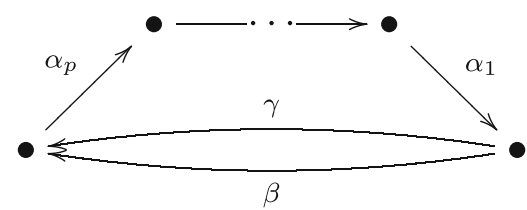

bound by $\alpha_{p} \beta, \alpha_{i} \alpha_{i+1}$ for $i \in[1, r]$, and $\gamma \alpha_{1}$. Moreover, for $p \in \mathbb{N}_{+}, \Lambda_{0}(p+1,-1)$ is the algebra of the quiver

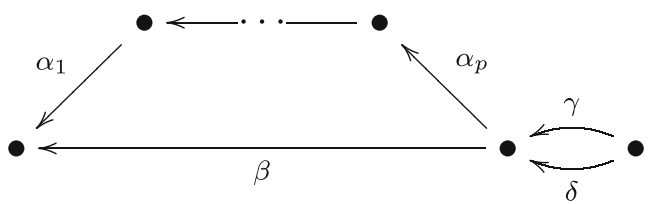

bound by $\alpha_{p} \gamma$ and $\beta \delta$. Furthermore, for $p_{1}, p_{2} \in \mathbb{N}_{+}, p_{3}, p_{4} \in \mathbb{N}$, and $r_{1} \in\left[0, p_{1}-1\right]$, such that $p_{2}+p_{3} \geq 2$ and $p_{4}+r_{1} \geq 1, \Lambda_{1}\left(p_{1}, p_{2}, p_{3}, p_{4}, r_{1}\right)$ is the algebra of the quiver

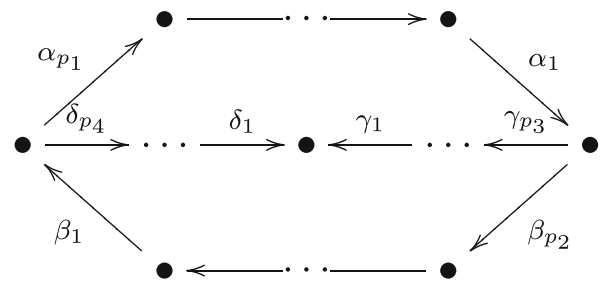


bound by $\alpha_{i} \alpha_{i+1}$ for $i \in\left[p_{1}-r_{1}, p_{1}-1\right], \alpha_{p_{1}} \beta_{1}, \beta_{i} \beta_{i+1}$ for $i \in\left[1, p_{2}-1\right]$, and $\beta_{p_{2}} \alpha_{1}$. Finally, for $p_{1}, p_{2} \in \mathbb{N}_{+}, p_{3} \in \mathbb{N}, r_{1} \in\left[0, p_{1}-1\right]$, and $r_{2} \in\left[0, p_{2}-1\right]$, such that $p_{3}+r_{1}+r_{2} \geq 1, \Lambda_{2}\left(p_{1}, p_{2}, p_{3}, r_{1}, r_{2}\right)$ is the algebra of the quiver

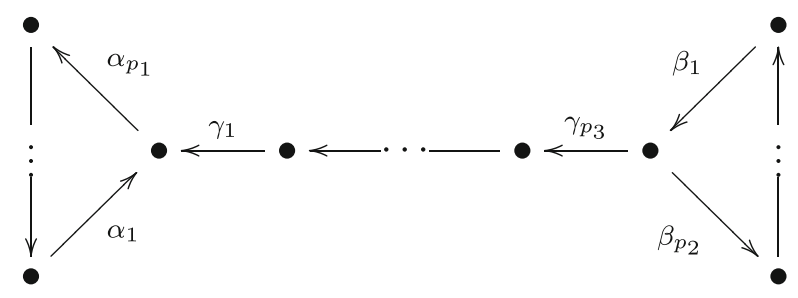

bound by $\alpha_{i} \alpha_{i+1}$ for $i \in\left[p_{1}-r_{1}, p_{1}-1\right], \alpha_{p_{1}} \alpha_{1}, \beta_{i} \beta_{i+1}$ for $i \in\left[p_{2}-r_{2}, p_{2}-1\right]$, and $\beta_{p_{2}} \beta_{1}$.

The main aim of this paper is to prove the following theorem.

Theorem A The above defined algebras are representatives of the derived equivalence classes of the gentle two-cycle algebras. More precisely,

(1) if $\Lambda$ is a gentle two-cycle algebra, then $\Lambda$ is derived equivalent to one of the above defined algebras, and

(2) the above defined algebras are pairwise not derived equivalent.

Parts of Theorem A have been already proved in [17] (see also [7]). More precisely, the following claims have been proved there:

(1) If $\Lambda$ is a gentle two-cycle algebra, then $\Lambda$ is derived equivalent to an algebra from one of the families $\Lambda_{0}, \Lambda_{1}$ and $\Lambda_{2}$.

(2) The algebras from different families are not derived equivalent.

(3) The algebras from family $\Lambda_{1}\left(\Lambda_{2}\right)$ are pairwise not derived equivalent.

Thus in order to prove Theorem A, we have to show the following.

Theorem B If $p^{\prime}, p^{\prime \prime} \in \mathbb{N}_{+}, r^{\prime} \in\left[-1, p^{\prime}-1\right], r^{\prime \prime} \in\left[-1, p^{\prime \prime}-1\right]$, and $\left(p^{\prime}, r^{\prime}\right) \neq(1,-1) \neq$ $\left(p^{\prime \prime}, r^{\prime \prime}\right)$, then the algebras $\Lambda_{0}\left(p^{\prime}, r^{\prime}\right)$ and $\Lambda_{0}\left(p^{\prime \prime}, r^{\prime \prime}\right)$ are not derived equivalent.

Partial versions of Theorem B have been obtained independently by Amiot [1] and Kalck [33]. In particular, Amiot has proved this result in the case when $r$ 's are "small" relative to $p$ 's (see Proposition 2.3 for a precise statement) by refining her earlier joint results with Grimeland on surface algebras [2]. The new ingredient of the paper is Corollary 3.2, which says that if $\Lambda\left(p, r^{\prime}\right)$ and $\Lambda\left(p, r^{\prime \prime}\right)$ are derived equivalent, then $\Lambda\left(p+1, r^{\prime}\right)$ and $\Lambda\left(p+1, r^{\prime \prime}\right)$ are derived equivalent. Using this and induction we reduce the situation to the setup of Amiot's result.

We note that one can replace derived equivalence by tilting-cotilting equivalence (see for example [6]) in Theorems A and B. Indeed, obviously if algebras are not derived equivalent, then they are not tilting-cotilting equivalent. On the other hand, every derived equivalence obtained in [17] is realized via a tilting-cotilting equivalence.

The paper consists of two sections. In Section 2 we recall necessary tools, including the invariant of Avella-Alaminos and Geiss, Auslander-Reiten quivers, (generalized APR) reflections, and behavior of derived equivalences under one-point coextensions. Next in 
Section 3 we prove Theorem B. In the paper we use a formalism of bound quivers introduced by Gabriel [23]. For related background see for example [5].

The author would like to thank the referee for the remarks, which helped improve the paper significantly. The author was supported by the National Science Center Grant No. 2015/17/B/ST1/01731.

\section{Preliminaries}

\subsection{Quivers and Their Representations}

By a quiver $\Delta$ we mean a set $\Delta_{0}$ of vertices and a set $\Delta_{1}$ of arrows together with two maps $s=s_{\Delta}, t=t_{\Delta}: \Delta_{1} \rightarrow \Delta_{0}$, which assign to $\alpha \in \Delta_{1}$ the starting vertex $s \alpha$ and the terminating vertex $t \alpha$, respectively. We assume that all considered quivers $\Delta$ are locally finite, i.e. for each $x \in \Delta_{0}$ there is only a finite number of $\alpha \in \Delta_{1}$ such that either $s \alpha=x$ or $t \alpha=x$. A quiver $\Delta$ is called finite if $\Delta_{0}$ (and, consequently, also $\Delta_{1}$ ) is a finite set. For technical reasons we assume that if $\Delta$ is a quiver, then $\Delta_{0} \neq \emptyset$ and $\Delta$ has no isolated vertices, i.e. there is no $x \in \Delta_{0}$ such that $s \alpha \neq x \neq t \alpha$ for each $\alpha \in \Delta_{1}$. In particular, $\Delta_{1} \neq \emptyset$.

Let $\Delta$ be a quiver. If $l \in \mathbb{N}_{+}$, then by a path in $\Delta$ of length $l$ we mean every sequence $\sigma=\alpha_{1} \cdots \alpha_{l}$ such that $\alpha_{i} \in \Delta_{1}$ for each $i \in[1, l]$ and $s \alpha_{i}=t \alpha_{i+1}$ for each $i \in[1, l-1]$. In the above situation we put $s \sigma:=s \alpha_{l}$ and $t \sigma:=t \alpha_{1}$. Moreover, we call $\alpha_{1}$ and $\alpha_{l}$ the terminating and the starting arrows of $\sigma$, respectively. Observe that each $\alpha \in \Delta$ is a path in $\Delta$ of length 1 . Moreover, for each $x \in \Delta_{0}$ we introduce the path $\mathbf{1}_{x}$ in $\Delta$ of length 0 such that $s \mathbf{1}_{x}:=x=: t \mathbf{1}_{x}$. We denote the length of a path $\sigma$ by $\ell(\sigma)$. If $\sigma^{\prime}$ and $\sigma^{\prime \prime}$ are two paths in $\Delta$ such that $s \sigma^{\prime}=t \sigma^{\prime \prime}$, then we define the composition $\sigma^{\prime} \sigma^{\prime \prime}$ of $\sigma^{\prime}$ and $\sigma^{\prime \prime}$, which is a path in $\Delta$ of length $\ell\left(\sigma^{\prime}\right)+\ell\left(\sigma^{\prime \prime}\right)$, in the obvious way (in particular, $\sigma \mathbf{1}_{s \sigma}=\sigma=\mathbf{1}_{t \sigma} \sigma$ for each path $\sigma$ ). A path $\sigma_{0}$ is called a subpath of a path $\sigma$, if there exist paths $\sigma^{\prime}$ and $\sigma^{\prime \prime}$ such that $\sigma=\sigma^{\prime} \sigma_{0} \sigma^{\prime \prime}$.

By a (monomial) bound quiver we mean a pair $\Lambda=(\Delta, R)$ consisting of a finite quiver $\Delta$ and a set $R$ of paths in $\Delta$, such that:

(1) $\ell(\rho)>1$ for each $\rho \in R$, and

(2) there exists $n \in \mathbb{N}_{+}$such that every path $\sigma$ in $\Delta$ with $\ell(\sigma)=n$ has a subpath which belongs to $R$.

If $\Lambda=(\Delta, R)$ is a bound quiver, then by a path in $\Lambda$ we mean a path in $\Delta$ which does not have a subpath from $R$. A path $\sigma$ in $\Lambda$ is said to be maximal in $\Lambda$ if $\sigma$ is not a subpath of a longer path in $\Lambda$. The lack of isolated vertices in $\Delta$ implies that $\ell(\sigma)>0$ for each maximal path $\sigma$ in $\Lambda$.

By a representation $V$ of a bound quiver $\Lambda=(\Delta, R)$ we mean a collection of finitedimensional vector spaces $V_{x}, x \in \Delta_{0}$, and linear maps $V_{\alpha}: V_{s \alpha} \rightarrow V_{t \alpha}, \alpha \in \Delta_{1}$, such that the induced map $V_{\rho}: V_{s \rho} \rightarrow V_{t \rho}$ is zero for every $\rho \in R$. If $V$ and $W$ are representations, then a homomorphism $f: V \rightarrow W$ is a collection of linear maps $f_{x}: V_{x} \rightarrow W_{x}, x \in \Delta_{0}$, such that $f_{t \alpha} V_{\alpha}=W_{\alpha} f_{s \alpha}$ for every arrow $\alpha$ in $\Delta$. The category rep $\Lambda$ of representations of $\Lambda$ is an abelian category. We call bound quivers $\Lambda^{\prime}$ and $\Lambda^{\prime \prime}$ derived equivalent (and write $\left.\Lambda^{\prime} \simeq_{\text {der }} \Lambda^{\prime \prime}\right)$, if the derived categories $\mathcal{D}^{b}\left(\operatorname{rep} \Lambda^{\prime}\right)$ and $\mathcal{D}^{b}\left(\operatorname{rep} \Lambda^{\prime \prime}\right)$ are triangle equivalent. We will usually write shortly $\mathcal{D}^{b}(\Lambda)$ instead of $\mathcal{D}^{b}(\operatorname{rep} \Lambda)$ if $\Lambda$ is a bound quiver.

A connected bound quiver $\Lambda=(\Delta, R)$ is called gentle if the following conditions are satisfied: 
(1) $R$ consists of paths of length 2 ,

(2) for each $x \in \Delta_{0}$ there are at most two $\alpha \in \Delta_{1}$ such that $s \alpha=x$ and at most two $\alpha \in \Delta_{1}$ such that $t \alpha=x$,

(3) for each $\alpha \in \Delta_{1}$ there is at most one $\alpha^{\prime} \in \Delta_{1}$ such that $s \alpha^{\prime}=t \alpha$ and $\alpha^{\prime} \alpha \notin R$, and at most one $\alpha^{\prime} \in \Delta_{1}$ such that $t \alpha^{\prime}=s \alpha$ and $\alpha \alpha^{\prime} \notin R$,

(4) for each $\alpha \in \Delta_{1}$ there is at most one $\alpha^{\prime} \in \Delta_{1}$ such that $s \alpha^{\prime}=t \alpha$ and $\alpha^{\prime} \alpha \in R$, and at most one $\alpha^{\prime} \in \Delta_{1}$ such that $t \alpha^{\prime}=s \alpha$ and $\alpha \alpha^{\prime} \in R$.

Let $\Lambda=(\Delta, R)$ be a gentle bound quiver. Note that by condition (1) above a path $\alpha_{1} \ldots \alpha_{l}$ in $\Delta$ is a path in $\Lambda$ if and only if $\alpha_{i} \alpha_{i+1} \notin R$ for all $i \in[1, l-1]$. We call a path $\alpha_{1} \ldots \alpha_{l}$ in $\Delta$ an antipath in $\Lambda$ if $\alpha_{i} \alpha_{i+1} \in R$ for all $i \in[1, l-1]$. In particular, every path of length at most 1 is an antipath. Again we call an antipath $\omega$ maximal if $\omega$ is not a subpath of a longer antipath in $\Lambda$.

\subsection{The Invariant of Avella-Alaminos and Geiss}

Throughout this subsection $\Lambda=(\Delta, R)$ is a fixed gentle bound quiver.

By a permitted thread in $\Lambda$ we mean either a maximal path in $\Lambda$ or $\mathbf{1}_{x}$, for $x \in \Delta_{0}$, such that there is at most one arrow $\alpha$ with $s \alpha=x$, there is at most one arrow $\beta$ with $t \beta=x$, and if such $\alpha$ and $\beta$ exist, then $\alpha \beta \notin R$. Similarly, by a forbidden thread we mean either a maximal antipath in $\Lambda$ or $\mathbf{1}_{x}$, for $x \in \Delta_{0}$, such that there is at most one arrow $\alpha$ with $s \alpha=x$, there is at most one arrow $\beta$ with $t \beta=x$, and if such $\alpha$ and $\beta$ exist, then $\alpha \beta \in R$.

Denote by $\mathcal{P}$ and $\mathcal{F}$ the sets of the permitted and forbidden threads in $\Lambda$, respectively. We define bijections $\Phi_{1}: \mathcal{P} \rightarrow \mathcal{F}$ and $\Phi_{2}: \mathcal{F} \rightarrow \mathcal{P}$. First, if $\sigma$ is a maximal path in $\Lambda$, then we put $\Phi_{1}(\sigma):=\omega$, where $\omega$ is the unique forbidden thread such that $t \omega=t \sigma$ and either $\ell(\omega)=0$ or $\ell(\omega)>0$ and the terminating arrows of $\sigma$ and $\omega$ differ. If $\mathbf{1}_{x}$, for $x \in \Delta_{0}$, is a permitted thread, there are two cases to consider. If there is an arrow $\beta$ such that $t \beta=x$ (note that such $\beta$ is uniquely determined), then $\Phi_{1}\left(\mathbf{1}_{x}\right)$ is the (unique) forbidden thread whose terminating arrow is $\beta$. Otherwise we put $\Phi_{1}\left(\mathbf{1}_{x}\right):=\mathbf{1}_{x}$. We define $\Phi_{2}$ dually. Namely, if $\omega$ is a maximal antipath, then $\Phi_{2}(\omega):=\sigma$, where $\sigma$ is the permitted thread such that $s \sigma=s \omega$ and either $\ell(\sigma)=0$ or $\ell(\sigma)>0$ and the starting arrows of $\omega$ and $\sigma$ differ. Now, let $x \in \Delta_{0}$ and $\mathbf{1}_{x}$ be a forbidden thread. If there is $\alpha \in \Delta_{1}$ such that $s \alpha=x$, then $\Phi_{2}\left(\mathbf{1}_{x}\right)$ is the permitted thread whose starting arrow is $\alpha$. Otherwise, $\Phi_{2}\left(\mathbf{1}_{x}\right):=\mathbf{1}_{x}$. Finally, we put $\Phi:=\Phi_{1} \Phi_{2}: \mathcal{F} \rightarrow \mathcal{F}$.

Let $\mathcal{F}^{\prime}$ be the set of arrows in $\Delta$ which are not subpaths of any maximal antipath in $\Lambda$ (i.e. every antipath containing $\alpha$ can be extended to a longer antipath). For every $\alpha \in \mathcal{F}^{\prime}$ there exists uniquely determined $\alpha^{\prime} \in \mathcal{F}^{\prime}$ such that $\alpha \alpha^{\prime} \in R$. We put $\Phi^{\prime}(\alpha):=\alpha^{\prime}$. In this way we get a bijection $\Phi^{\prime}: \mathcal{F}^{\prime} \rightarrow \mathcal{F}^{\prime}$. In other words, $\mathcal{F}^{\prime}$ is the set of arrows which lie on oriented cycles with full relations. Moreover, two arrows in $\mathcal{F}^{\prime}$ belong to the same orbit with respect to the action of $\Phi^{\prime}$ if and only if they lie on the same oriented cycle with full relations.

The following result seems to be well-known, however we could not find a reference for it, hence we include its proof for completeness.

Proposition 2.1 Let $\Lambda=(\Delta, R)$ be a gentle bound quiver. Then gldim $\Lambda<\infty$ if and only if $\mathcal{F}^{\prime}=\emptyset$.

Proof For a vertex $x$ of $\Delta$ we denote by $S_{x}$ and $P_{x}$ the simple and the projective representations of $\Lambda$ at $x$, respectively. For $\alpha \in \Delta_{1}$ we denote by $P_{\alpha}$ the corresponding map $P_{t \alpha} \rightarrow P_{s \alpha}$. 
Assume first $\mathcal{F}^{\prime}=\varnothing$ and fix $x \in \Delta_{0}$. Assume there are exactly two arrows $\alpha$ and $\beta$ starting at $x$. Let $\alpha_{n} \cdots \alpha_{1}$ and $\beta_{m} \cdots \beta_{1}$ be the maximal antipaths, whose starting arrows are $\alpha$ and $\beta$, respectively (in particular, $\alpha_{1}=\alpha$ and $\beta_{1}=\beta$ ) - such antipaths exist, since $\mathcal{F}^{\prime}=\emptyset$. Then

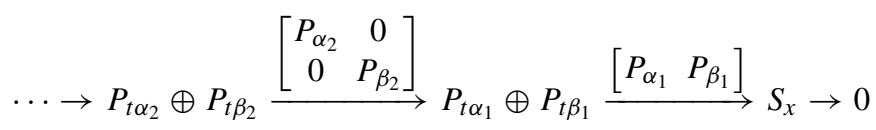

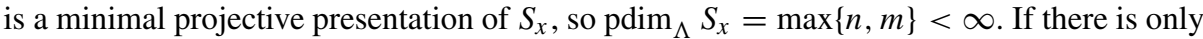
one arrow starting at $x$, then we have a degenerate version of the above. Finally, if there is no arrow starting at $x$, then $S_{x}=P_{x}$.

Now assume $\mathcal{F}^{\prime} \neq \emptyset$, choose $\alpha \in \mathcal{F}^{\prime}$, and put $\alpha_{i}:=\Phi^{\prime-i}(\alpha), i \in \mathbb{N}$. Then

$$
\cdots \rightarrow P_{t \alpha_{1}} \stackrel{P_{\alpha_{1}}}{\longrightarrow} P_{t \alpha_{0}} \stackrel{P_{\alpha_{0}}}{\longrightarrow} P_{s \alpha} \rightarrow \text { Coker } P_{\alpha} \rightarrow 0
$$

is a minimal projective presentation of Coker $P_{\alpha}$, so $\operatorname{pdim}_{\Lambda}$ Coker $P_{\alpha}=\infty$.

Let $\mathcal{F} / \Phi$ be the set of orbits in $\mathcal{F}$ with respect to the action of $\Phi$. For each $\mathcal{O} \in \mathcal{F} / \Phi$ we put $n_{\mathcal{O}}:=|\mathcal{O}|$ and $m_{\mathcal{O}}:=\sum_{\omega \in \mathcal{O}} \ell(\omega)$. Similarly, if $\mathcal{O} \in \mathcal{F}^{\prime} / \Phi^{\prime}$, then $n_{\mathcal{O}}:=0$ and $m_{\mathcal{O}}:=|\mathcal{O}|$. We define $\phi_{\Lambda}: \mathbb{N}^{2} \rightarrow \mathbb{N}$ by the formula:

$$
\phi_{\Lambda}(n, m):=\left|\left\{\mathcal{O} \in \mathcal{F} / \Phi \cup \mathcal{F}^{\prime} / \Phi^{\prime}:\left(n_{\mathcal{O}}, m_{\mathcal{O}}\right)=(n, m)\right\}\right| \quad(n, m \in \mathbb{N}) .
$$

Avella-Alaminos and Geiss have proved [8] that $\phi_{\Lambda}$ is a derived invariant, i.e. if $\Lambda^{\prime}$ and $\Lambda^{\prime \prime}$ are derived equivalent gentle bound quivers, then $\phi_{\Lambda^{\prime}}=\phi_{\Lambda^{\prime \prime}}$.

For a function $\phi: \mathbb{N}^{2} \rightarrow \mathbb{N}$ we put $\|\phi\|:=\sum_{(n, m) \in \mathbb{N}^{2}} \phi(n, m)$. If $\Lambda$ is a gentle bound quiver, then $\left\|\phi_{\Lambda}\right\|$ equals $|\mathcal{F} / \Phi|+\left|\mathcal{F}^{\prime} / \Phi^{\prime}\right|$. We will need the following observation.

Lemma 2.2 Let $\Lambda=(\Delta, R)$ be a gentle bound quiver such that $\left\|\phi_{\Lambda}\right\|=1$. Then $\mathcal{F}^{\prime}=\emptyset$, hence gldim $\Lambda<\infty$. Moreover, if $\mathcal{O} \in \mathcal{F} / \Phi$, then $n_{\mathcal{O}} \neq m_{\mathcal{O}}$.

Proof Let $\mathcal{O}$ be the unique element of $\mathcal{F} / \Phi \cup \mathcal{F}^{\prime} / \Phi^{\prime}$ (i.e. either $\mathcal{O}=\mathcal{F}$ or $\mathcal{O}=\mathcal{F}^{\prime}$ ). It follows from [15, Lemma 3.2], that $n_{\mathcal{O}}=2\left|\Delta_{0}\right|-\left|\Delta_{1}\right|$ and $m_{\mathcal{O}}=\left|\Delta_{1}\right|$. If $\mathcal{O}=\mathcal{F}^{\prime}$, then $n_{\mathcal{O}}=0$, hence $\left|\Delta_{1}\right|=2\left|\Delta_{0}\right|$. By condition (2) of the definition of a gentle bound quiver this means that for each $x \in \Delta_{0}$ there are exactly two arrows starting at $x$. Consequently, condition (4) of the definition implies that for each $\alpha \in \Delta_{1}$ there exists $\alpha^{\prime} \in \Delta_{1}$ such that $s \alpha^{\prime}=t \alpha$ and $\alpha^{\prime} \alpha \notin R$. Thus, there exist paths in $\Lambda$ of arbitrary length, which contradicts condition (2) of the definition of a bound quiver. Consequently, $\mathcal{O}=\mathcal{F}$, hence $\mathcal{F}^{\prime}=\emptyset$.

Now assume $n_{\mathcal{O}}=m_{\mathcal{O}}$. Then $2\left|\Delta_{0}\right|-\left|\Delta_{1}\right|=\left|\Delta_{1}\right|$, i.e. $\left|\Delta_{0}\right|=\left|\Delta_{1}\right|$, hence $\Lambda$ is a onecycle gentle bound quiver. However in this case $\left\|\phi_{\Lambda}\right\|=2$ (see [8, Section 7]), hence the claim follows.

\subsection{Boundary Complexes}

Let $\Lambda=(\Delta, R)$ be a gentle bound quiver. One defines the Auslander-Reiten quiver $\Gamma\left(\mathcal{D}^{b}(\Lambda)\right)$ of $\mathcal{D}^{b}(\Lambda)$ in the following way: the vertices of $\Gamma\left(\mathcal{D}^{b}(\Lambda)\right)$ are (representatives of) the isomorphism classes of the indecomposable complexes in $\mathcal{D}^{b}(\Lambda)$ and the number of arrows between vertices $X$ and $Y$ equals the dimension of the space of irreducible maps between $X$ and $Y$. 
Since the gentle bound quivers are Gorenstein (see [27]), the Auslander-Reiten translation $\tau$ (see [30]) is an autoequivalence on the subcategory of perfect complexes (i.e. complexes, which are quasi-isomorphic to bounded complexes of projective representations). In particular, if gldim $\Lambda<\infty$, then $\tau$ is an automorphism of $\mathcal{D}^{b}(\Lambda)$.

An indecomposable complex $X \in \mathcal{D}^{b}(\Lambda)$ is called boundary if $X$ is perfect and there is only one arrow in $\Gamma\left(\mathcal{D}^{b}(\Lambda)\right)$ terminating at $X$. Equivalently, $X$ is perfect and in the Auslander-Reiten triangle (see [30]) terminating at $X$ the middle term is indecomposable.

The invariant of Avella-Alaminos and Geiss describes the action of the shift $\Sigma$ on the components of $\Gamma\left(\mathcal{D}^{b}(\Lambda)\right)$ containing boundary complexes. We will use the following excerpt from their results in [8, Sections 5 and 6]. First, there exist homogeneous tubes in $\Gamma\left(\mathcal{D}^{b}(\Lambda)\right)$ if and only if there exists an orbit $\mathcal{O} \in \mathcal{F} / \Phi$ such that $n_{\mathcal{O}}=1=m_{\mathcal{O}}$. Let $\mathcal{C}$ be the family of components of $\Gamma\left(\mathcal{D}^{b}(\Lambda)\right)$, which contain boundary complexes, but are not homogeneous tubes. If $\mathcal{C} / \Sigma$ is the set of orbits in $\mathcal{C}$ with respect to the action of $\Sigma$ and $\mathcal{X}$ is the set of orbits $\mathcal{O} \in \mathcal{F} / \Phi$ such that $\left(n_{\mathcal{O}}, m_{\mathcal{O}}\right) \neq(1,1)$, then $|\mathcal{C} / \Sigma|=|\mathcal{X}|$. In particular, if $|\mathcal{X}|=1$ and $X$ and $Y$ are boundary complexes, which do not lie in homogeneous tubes, then there exists $p \in \mathbb{Z}$ such that $\Sigma^{p} X$ and $Y$ belong to the same component. If $\left\|\phi_{\Lambda}\right\|=1$, we have even more.

Lemma 2.3 Let $\Lambda$ be a gentle bound quiver such that $\left\|\phi_{\Lambda}\right\|=1$. If $X$ and $Y$ are boundary complexes in $\mathcal{D}^{b}(\Lambda)$, then there exists an autoequivalence $F$ of $\mathcal{D}^{b}(\Lambda)$ such that $F X=Y$.

Proof Assume first that $\Lambda$ is derived equivalent to a hereditary algebra of Dynkin type $\mathbb{A}$, i.e. $\Lambda$ is a gentle tree. In this case $\Gamma\left(\mathcal{D}^{b}(\Lambda)\right)$ is $\mathbb{Z} \mathbb{A}_{n}$ for some $n \in \mathbb{N}_{+}$(see [29, Section I.5]), hence the boundary complexes form two orbits with respect to the action of $\tau$, which is an autoequivalence of $\mathcal{D}^{b}(\Lambda)$, since gldim $\Lambda<\infty$ by Lemma 2.2. Moreover, $\Sigma$ interchanges these orbits, hence the claim follows in this case.

If $\Lambda$ is one-cycle gentle bound quiver, then $\left\|\phi_{\Lambda}\right\|=2 \neq 1$ by [8, Section 7], hence we may assume $\Lambda$ is not of polynomial growth by [39, Theorem 1.1]. Let $\mathcal{O}$ be the unique element of $\mathcal{F} / \Phi \cup \mathcal{F}^{\prime} / \Phi^{\prime}$. Lemma 2.2 implies that $\mathcal{O} \in \mathcal{F} / \Phi$ and $\left(n_{\mathcal{O}}, m_{\mathcal{O}}\right) \neq(1,1)$. In particular, there are no homogeneous tubes in $\Gamma\left(\mathcal{D}^{b}(\Lambda)\right)$. Consequently, by the discussion above we know there exists $p \in \mathbb{Z}$ such that $\Sigma^{p} X$ and $Y$ belong to the same component of $\Gamma\left(\mathcal{D}^{b}(\Lambda)\right)$. Moreover, [26, Theorem 2.6] implies that $\Sigma^{p} X$ and $Y$ belong to the same $\tau$ orbit, i.e. there exists $q \in \mathbb{Z}$ such that $\tau^{q} \Sigma^{p} X=Y$. Finally, gldim $\Lambda<\infty$ by Lemma 2.2, hence $\tau$ is an autoequivalence of $\mathcal{D}^{b}(\Lambda)$, and the claim follows.

If $\sigma$ is a path in $\Lambda$, then we have the corresponding (string) representation $M(\sigma)$ (see for example [22]). We have the following observation.

Lemma 2.4 Let $\Lambda$ be a gentle bound quiver. If $\sigma$ is a maximal path in $\Lambda$, then $M(\sigma)$ (viewed as a complex concentrated in degree 0 ) is a boundary complex in $\mathcal{D}^{b}(\Lambda)$.

Proof In the terminology of [14] (see also [12]) a projective presentation of $M(\sigma)$ is given by the complex which corresponds to the antipath $\Phi_{2}^{-1}(\sigma)$. In particular, this implies that $M(\sigma)$ is a perfect complex in $\mathcal{D}^{b}(\Lambda)$. Moreover, if one uses results of [14] in order to calculate the Auslander-Reiten triangle terminating at $M(\sigma)$, then one gets that its middle term is indecomposable. Alternatively, one may use the Happel functor [28, 29] and wellknown formulas (see for example [22, 41]) for calculating the Auslander-Reiten triangles in the stable category of the category of representations of the repetitive category $\hat{\Lambda}$ of $\Lambda$. We leave details to the reader. 
We formulate the following consequence.

Corollary 2.5 Let $\Lambda^{\prime}$ and $\Lambda^{\prime \prime}$ be derived equivalent gentle bound quivers such that $\left\|\phi_{\Lambda^{\prime}}\right\|=$ $1=\left\|\phi_{\Lambda^{\prime \prime}}\right\|$. If $\sigma^{\prime}$ and $\sigma^{\prime \prime}$ are maximal paths in $\Lambda^{\prime}$ and $\Lambda^{\prime \prime}$, respectively, then there exists a derived equivalence $F: \mathcal{D}^{b}\left(\Lambda^{\prime}\right) \rightarrow \mathcal{D}^{b}\left(\Lambda^{\prime \prime}\right)$ such that $F\left(M\left(\sigma^{\prime}\right)\right)=M\left(\sigma^{\prime \prime}\right)$.

Proof Let $G: \mathcal{D}^{b}\left(\Lambda^{\prime}\right) \rightarrow \mathcal{D}^{b}\left(\Lambda^{\prime \prime}\right)$ be a derived equivalence. We know from Lemma 2.4 that $M\left(\sigma^{\prime}\right)$ and $M\left(\sigma^{\prime \prime}\right)$ are boundary complexes in $\mathcal{D}^{b}\left(\Lambda^{\prime}\right)$ and $\mathcal{D}^{b}\left(\Lambda^{\prime \prime}\right)$, respectively. Consequently, $G\left(M\left(\sigma^{\prime}\right)\right)$ and $M\left(\sigma^{\prime \prime}\right)$ are boundary complexes in $\mathcal{D}^{b}\left(\Lambda^{\prime \prime}\right)$. Thus, by Lemma 2.3 , there exists an autoequivalence $H$ of $\mathcal{D}^{b}\left(\Lambda^{\prime \prime}\right)$ such that $H\left(G\left(M\left(\sigma^{\prime}\right)\right)\right)=M\left(\sigma^{\prime \prime}\right)$. We take $F=H \circ G$.

\subsection{One-point Coextensions}

If $\Lambda$ is a bound quiver and $M$ is a representation of $\Lambda$, then one defines a bound quiver $[M] \Lambda$, called the one-point coextension of $\Lambda$ by $M$ (see for example [9]). However, usually $[M] \Lambda$ is not monomial, even if $\Lambda$ is. Consequently, in the paper we only consider one-point coextensions of the form $[M(\sigma)] \Lambda$, where $\Lambda$ is a gentle bound quiver and $\sigma$ is a maximal path in $\Lambda$.

Let $\Lambda=(\Delta, R)$ be a gentle bound quiver and $\sigma$ a maximal path in $\Lambda$. We define the one-point coextension $[M(\sigma)] \Lambda$ of $\Lambda$ by $M(\sigma)$ as follows: $[M(\sigma)] \Lambda:=\left(\Delta^{\prime}, R^{\prime}\right)$, where

(1) $\Delta^{\prime}$ is obtained from $\Delta$ by adding a new arrow $\alpha$ starting at $t \sigma$ and terminating at a new vertex $x$;

(2) if there exists (necessarily unique) arrow $\alpha^{\prime}$ in $\Delta$, which terminates at $t \sigma$, but is not the terminating arrow of $\sigma$, then $R^{\prime}:=R \cup\left\{\alpha \alpha^{\prime}\right\}$; otherwise, $R^{\prime}:=R$.

We write shortly $[\sigma] \Lambda$ instead of $[M(\sigma)] \Lambda$. One easily gets the following.

Lemma 2.6 Let $\Lambda$ be gentle bound quiver. If $\sigma$ is a maximal path in $\Lambda$, then $[\sigma] \Lambda$ is a gentle bound quiver.

Proof Exercise.

The following is a special version of the dual of Barot and Lenzing's [9, Theorem 1].

Proposition 2.7 Let $\sigma^{\prime}$ and $\sigma^{\prime \prime}$ be maximal paths in gentle bound quivers $\Lambda^{\prime}$ and $\Lambda^{\prime \prime}$, respectively. If there exists a triangle equivalence $F: \mathcal{D}^{b}\left(\Lambda^{\prime}\right) \rightarrow \mathcal{D}^{b}\left(\Lambda^{\prime \prime}\right)$ such that $F\left(M\left(\sigma^{\prime}\right)\right)=M\left(\sigma^{\prime \prime}\right)$, then $\left[\sigma^{\prime}\right] \Lambda^{\prime}$ and $\left[\sigma^{\prime \prime}\right] \Lambda^{\prime \prime}$ are derived equivalent.

Combining Proposition 2.7 with Corollary 2.5 we obtain.

Corollary 2.8 Let $\Lambda^{\prime}$ and $\Lambda^{\prime \prime}$ be derived equivalent gentle bound quivers such that $\left\|\phi_{\Lambda^{\prime}}\right\|=$ $1=\left\|\phi_{\Lambda^{\prime \prime}}\right\|$. If $\sigma^{\prime}$ and $\sigma^{\prime \prime}$ are maximal paths in $\Lambda^{\prime}$ and $\Lambda^{\prime \prime}$, respectively, then $\left[\sigma^{\prime}\right] \Lambda^{\prime}$ and $\left[\sigma^{\prime \prime}\right] \Lambda^{\prime \prime}$ are derived equivalent.

\subsection{Reflections}

Let $\Lambda=(\Delta, R)$ be a gentle bound quiver. Let $x$ be a vertex in $\Delta$ such that there is no $\alpha \in \Delta_{1}$ with $s \alpha=x=t \alpha$ and for each $\alpha \in \Delta_{1}$ with $s \alpha=x$ there exists $\beta_{\alpha} \in \Delta_{1}$ with 
$t \beta_{\alpha}=x$ and $\alpha \beta_{\alpha} \notin R$. We define a bound quiver $\Lambda^{\prime}=\left(\Delta^{\prime}, R^{\prime}\right)$ in the following way: $\Delta_{0}^{\prime}=\Delta_{0}, \Delta_{1}^{\prime}=\Delta_{1}$,

$$
\begin{aligned}
& s_{\Delta^{\prime}} \alpha= \begin{cases}x & \text { if } t_{\Delta} \alpha=x, \\
s_{\Delta} \beta_{\alpha} & \text { if } s_{\Delta} \alpha=x, \\
s_{\Delta} \alpha & \text { otherwise }\end{cases} \\
& t_{\Delta^{\prime}} \alpha= \begin{cases}s_{\Delta} \alpha & \text { if } t_{\Delta} \alpha=x \\
x & \text { if there exists } \beta \in \Delta_{1} \text { such that } \\
t_{\Delta} \beta=x, s_{\Delta} \beta=t_{\Delta} \alpha \text { and } \beta \alpha \in R, & \text { otherwise, }\end{cases}
\end{aligned}
$$

and $R^{\prime}$ consists of the following relations:

- $\alpha \beta$, where $\alpha \beta \in R$ and $t_{\Delta} \alpha \neq x \neq s_{\Delta} \alpha$,

- $\alpha \beta_{\alpha}$, where $\alpha \in \Delta_{1}$ and $s_{\Delta} \alpha=x$,

- $\alpha \beta$, where $\alpha, \beta \in \Delta_{1}$ are such that $t_{\Delta} \alpha=x$ and $\gamma \beta \in R$ for some $\gamma \in \Delta_{1}, \gamma \neq \alpha$, with $t_{\Delta} \gamma=x$.

The following pictures, where the relations are indicated by dots, illustrate the situation: if locally (in a neighbourhood of $x$ ) $\Delta$ has the form

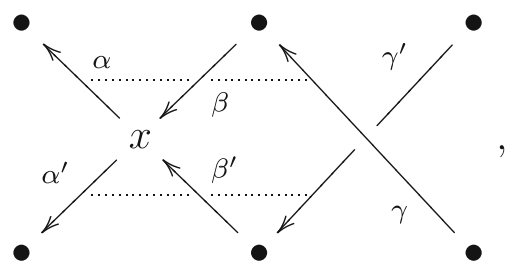

then locally $\Delta^{\prime}$ has the form

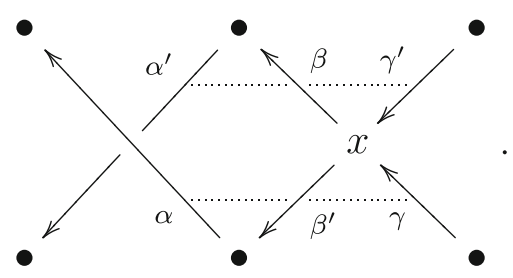

In the above situation we say that $\Lambda^{\prime}$ is obtained from $\Lambda$ by applying the (generalized APR) reflection at $x$. The bound quiver $\Lambda^{\prime}$ is derived equivalent to $\Lambda$ (see [17, Section 1]).

We will need the following application of this operation, which is a special version of [17, Lemma 1.1].

Lemma 2.9 Let $\Lambda=(\Delta, R)$ be a gentle bound quiver such that $\Delta$ is of the form

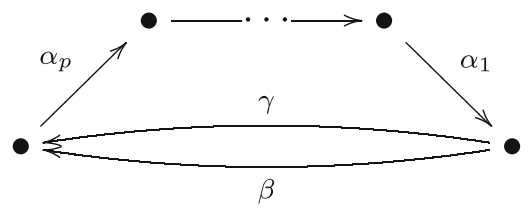


for $p \in \mathbb{N}_{+}$. Assume that $\alpha_{i-1} \alpha_{i} \notin R$ and $\alpha_{i} \alpha_{i+1} \in R$ for some $i \in[2, p-1]$. Then $\Lambda$ is derived equivalent to the gentle bound quiver $\Lambda^{\prime}:=\left(\Delta, R^{\prime}\right)$, where

$$
R^{\prime}:=\left(R \backslash\left\{\alpha_{i} \alpha_{i+1}\right\}\right) \cup\left\{\alpha_{i-1} \alpha_{i}\right\} .
$$

Proof We obtain $\Lambda^{\prime}$ from $\Lambda$ by applying the reflection at $t \alpha_{i}$, hence $\Lambda$ and $\Lambda^{\prime}$ are derived equivalent by the discussion above.

In the above situation we say that $\Lambda^{\prime}$ is obtained from $\Lambda$ by a shift of the relation $\alpha_{i} \alpha_{i+1}$.

\section{Proof of the Main Result}

The aim of this section is to prove that the bound quivers $\Lambda_{0}(p, r), p \in \mathbb{N}_{+}, r \in[-1, p+1]$, $(p, r) \neq(1,-1)$, are pairwise not derived equivalent. Observe (see also [17, Lemma 3.1]) that $\| \phi_{\Lambda_{0}(p, r) \|}=1$. The following observation is crucial.

Lemma 3.1 Let $p \in \mathbb{N}_{+}$and $r \in[-1, p-1]$, $(p, r) \neq(1,-1)$. If $\sigma$ is a maximal path in $\Lambda_{0}(p, r)$, then $[\sigma] \Lambda_{0}(p, r)$ is derived equivalent to $\Lambda_{0}(p+1, r)$.

Proof If $\sigma^{\prime}$ and $\sigma^{\prime \prime}$ are maximal paths in $\Lambda_{0}(p, r)$, then Corollary 2.8 implies that $\left[\sigma^{\prime}\right] \Lambda_{0}(p, r)$ and $\left[\sigma^{\prime \prime}\right] \Lambda_{0}(p, r)$ are derived equivalent. Thus it is enough to consider one particular $\sigma$.

First assume that $r \geq 0$ and let $\sigma$ be the maximal path whose terminating arrow is $\beta$, i.e. $\sigma:=\beta \alpha_{1}$, if $r>0$, and $\sigma:=\beta \alpha_{1} \cdots \alpha_{p} \gamma$, if $r=0$. Then $[\sigma] \Lambda_{0}(p, r)$ is the quiver

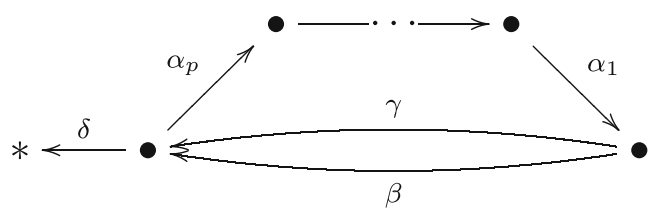

bound by relations $\alpha_{p} \beta, \alpha_{i} \alpha_{i+1}$ for $i \in[1, r], \gamma \alpha_{1}$ and $\delta \gamma$. If we apply the reflection at the vertex denoted by $*$, then we obtain the quiver

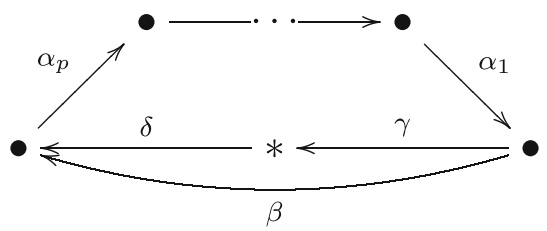

bound by relations $\alpha_{p} \beta, \alpha_{i} \alpha_{i+1}$ for $i \in[1, r]$, and $\gamma \alpha_{1}$. Now we apply again the reflection at the vertex denoted by $*$ and obtain the quiver

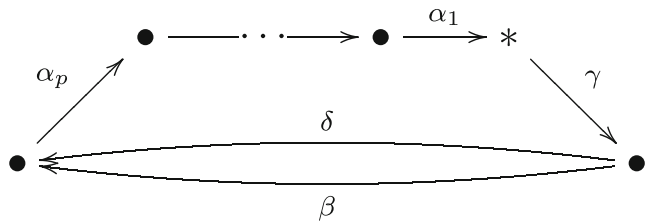


bound by relations $\alpha_{p} \beta, \alpha_{i} \alpha_{i+1}$ for $i \in[1, r]$, and $\delta \gamma$. Finally we shift relations (see Lemma 2.9) $r$ times and obtain (a bound quiver isomorphic to) $\Lambda_{0}(p+1, r)$.

We proceed similarly if $r=-1$. If $\sigma:=\beta \gamma$, then $[\sigma] \Lambda_{0}(p,-1)$ is the quiver

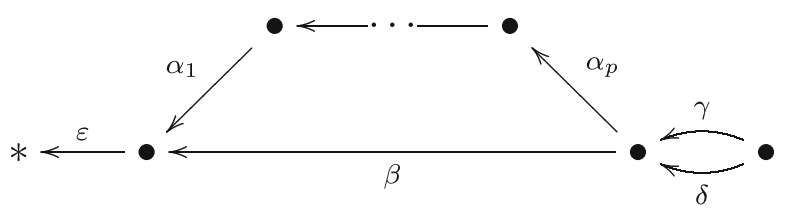

bound by relations $\alpha_{p} \gamma, \beta \delta$ and $\varepsilon \alpha_{1}$. By applying the reflection at the vertex denoted by $*$ we obtain $\Lambda_{0}(p+1,-1)$.

We have the following consequence of Lemma 3.1.

Corollary 3.2 Let $p \in \mathbb{N}_{+}$and $r^{\prime}, r^{\prime \prime} \in[-1, p-1],\left(p, r^{\prime}\right) \neq(1,-1) \neq\left(p, r^{\prime \prime}\right)$. If $\Lambda_{0}\left(p, r^{\prime}\right)$ and $\Lambda_{0}\left(p, r^{\prime \prime}\right)$ are derived equivalent, then $\Lambda_{0}\left(q, r^{\prime}\right)$ and $\Lambda_{0}\left(q, r^{\prime \prime}\right)$ are derived equivalent for all $q \geq p$.

Proof By induction it is enough to prove that $\Lambda_{0}\left(p+1, r^{\prime}\right)$ and $\Lambda_{0}\left(p+1, r^{\prime \prime}\right)$ are derived equivalent provided $\Lambda_{0}\left(p, r^{\prime}\right)$ and $\Lambda_{0}\left(p, r^{\prime \prime}\right)$ are derived equivalent. Let $\sigma^{\prime}$ and $\sigma^{\prime \prime}$ be maximal paths in $\Lambda_{0}\left(p, r^{\prime}\right)$ and $\Lambda_{0}\left(p, r^{\prime \prime}\right)$, respectively. Corollary 2.8 implies that $\left[\sigma^{\prime}\right] \Lambda_{0}\left(p, r^{\prime}\right)$ and $\left[\sigma^{\prime \prime}\right] \Lambda_{0}\left(p, r^{\prime \prime}\right)$ are derived equivalent. Since according to Lemma 3.1 $\left[\sigma^{\prime}\right] \Lambda_{0}\left(p, r^{\prime}\right) \simeq_{\operatorname{der}} \Lambda_{0}\left(p+1, r^{\prime}\right)$ and $\left[\sigma^{\prime \prime}\right] \Lambda_{0}\left(p, r^{\prime \prime}\right) \simeq_{\operatorname{der}} \Lambda_{0}\left(p+1, r^{\prime \prime}\right)$, the claim follows.

An important role in our proof is played by the following result due to Amiot [1, Corollary 4.4].

Proposition 3.3 Let $q \geq 3$ and $-1 \leq r^{\prime}, r^{\prime \prime} \leq \frac{q}{2}-1$. If $r^{\prime} \neq r^{\prime \prime}$, then the algebras $\Lambda_{0}\left(q, r^{\prime}\right)$ and $\Lambda_{0}\left(q, r^{\prime \prime}\right)$ are not derived equivalent.

Now we are ready to prove Theorem B.

Proof of Theorem B Let $p^{\prime}, p^{\prime \prime} \in \mathbb{N}, r^{\prime} \in\left[-1, p^{\prime}-1\right]$ and $r^{\prime \prime} \in\left[-1, p^{\prime \prime}-1\right]$ be such that $\left(p^{\prime}, r^{\prime}\right) \neq(1,-1) \neq\left(p^{\prime \prime}, r^{\prime \prime}\right)$. Obviously, $\Lambda_{0}\left(p^{\prime}, r^{\prime}\right)$ and $\Lambda_{0}\left(p^{\prime \prime}, r^{\prime \prime}\right)$ are not derived equivalent if $p^{\prime} \neq p^{\prime \prime}$ (e.g. they have different numbers of vertices). Thus assume that $p^{\prime}=p^{\prime \prime}$ and denote this common value by $p$. Choose $q \geq p$ such that $r^{\prime}, r^{\prime \prime} \leq \frac{q}{2}-1$. If $\Lambda_{0}\left(p, r^{\prime}\right)$ and $\Lambda_{0}\left(p, r^{\prime \prime}\right)$ are derived equivalent, then Corollary 3.2 implies that $\Lambda_{0}\left(q, r^{\prime}\right)$ and $\Lambda_{0}\left(q, r^{\prime \prime}\right)$ are derived equivalent as well. Consequently, $r^{\prime}=r^{\prime \prime}$ according to Proposition 3.3 and the claim follows.

Open Access This article is distributed under the terms of the Creative Commons Attribution 4.0 International License (http://creativecommons.org/licenses/by/4.0/), which permits unrestricted use, distribution, and reproduction in any medium, provided you give appropriate credit to the original author(s) and the source, provide a link to the Creative Commons license, and indicate if changes were made. 


\section{References}

1. Amiot, C.: The derived category of surface algebras: the case of torus with one boundary component. Algebr. Represent. Theory 19, 1059-1080 (2016)

2. Amiot, C., Grimeland, Y.: Derived invariants for surface algebras. J. Pure Appl. Algebra 220, 3133-3155 (2016)

3. Asashiba, H.: The derived equivalence classification of representation-finite selfinjective algebras. J. Algebra 214, 182-221 (1999)

4. Assem, I., Happel, D.: Generalized tilted algebras of type $A_{n}$. Comm. Algebra 9, 2101-2125 (1981)

5. Assem, I., Simson, D., Skowroński, A.: Elements of the representation theory of associative algebras. Vol. 1, London Mathematical Social Study Texts, p. x+458. Cambridge Univ. Press, Cambridge (2006)

6. Assem, I., Skowroński, A.: Iterated tilted algebras of type $\tilde{\mathbf{A}}_{n}$. Math. Z. 195, 269-290 (1987)

7. Avella-Alaminos, D.: Derived classification of gentle algebras with two cycles. Bol. Soc. Mat. Mexicana (3) 14, 177-216 (2008)

8. Avella-Alaminos, D., Geiss, Ch.: Combinatorial derived invariants for gentle algebras. J. Pure Appl. Algebra 212, 228-243 (2008)

9. Barot, M., Lenzing, H.: One-point extensions and derived equivalence. J. Algebra 264, 1-5 (2003)

10. Bastian, J.: Mutation classes of $\tilde{A}_{n}$-quivers and derived equivalence classification of cluster tilted algebras of type $\tilde{A}_{n}$. Algebra Number Theory 5, 567-594 (2011)

11. Beǐlinson, A.A.: Coherent sheaves on $\mathbf{P}^{n}$ and problems in linear algebra. Funktsional. Anal. i Prilozhen. 12, 68-69 (1978) (Russian); English transl., Funct. Anal. Appl. 12, 214-216 (1978)

12. Bekkert, V., Merklen, H.A.: Indecomposables in derived categories of gentle algebras. Algebr. Represent. Theory 6, 285-302 (2003)

13. Białkowski, J., Holm, T., Skowroński, A.: Derived equivalences for tame weakly symmetric algebras having only periodic modules. J. Algebra 269, 652-668 (2003)

14. Bobiński, G.: The almost split triangles for perfect complexes over gentle algebras. J. Pure Appl. Algebra 215, 642-654 (2011)

15. Bobiński, G., Buan, A.B.: The algebras derived equivalent to gentle cluster tilted algebras. J. Algebra Appl. 11, 1250012, 26 pp. (2012)

16. Bobiński, G., Geiß, Ch., Skowroński, A.: Classification of discrete derived categories. Cent. Eur. J. Math. 2, 19-49 (2004)

17. Bobiński, G., Malicki, P.: On derived equivalence classification of gentle two-cycle algebras. Colloq. Math. 112, 33-72 (2008)

18. Bocian, R., Holm, T., Skowroński, A.: Derived equivalence classification of one-parametric self-injective algebras. J. Pure Appl. Algebra 207, 491-536 (2006)

19. Brüstle, T.: Derived-tame tree algebras. Compositio Math. 129, 301-323 (2001)

20. Buan, A.B., Marsh, R., Reineke, M., Reiten, I., Todorov, G.: Tilting theory and cluster combinatorics. Adv. Math. 204, 572-618 (2006)

21. Buan, A.B., Vatne, D.F.: Derived equivalence classification for cluster-tilted algebras of type $A_{n}$. J. Algebra 319, 2723-2738 (2008)

22. Butler, M.C.R., Ringel, C.M.: Auslander-Reiten sequences with few middle terms and applications to string algebras. Comm. Algebra 15, 145-179 (1987)

23. Gabriel, P.: Unzerlegbare Darstellungen. I. Manuscripta Math. 6, 71-103; correction, ibid. 6 (1972), 309 (1972)

24. Geigle, W., Lenzing, H.: A class of weighted projective curves arising in representation theory of finitedimensional algebras, Singularities, Representation of Algebras, and Vector Bundles, Lecture Notes in Math., vol. 1273, pp. 265-297. Springer, Berlin (1987)

25. Geiss, Ch.: Derived tame algebras and Euler-forms. Math. Z. 239, 829-862 (2002). With an appendix by the author and B. Keller

26. Geiss, Ch., de la Peña, J.A.: Auslander-Reiten components for clans. Bol. Soc. Mat. Mexicana (3) 5(2), 307-326 (1999)

27. Geiss, Ch., Reiten, I. In: Buchweitz, R.-O., Lenzing, H. (eds.): Gentle algebras are gorenstein, Representations of algebras and related topics, pp. 129-133. Fields Institute Communications, vol. 45, American Mathematical Society, Providence, RI (2005)

28. Happel, D.: On the derived category of a finite-dimensional algebra. Comment. Math. Helv. 62, 339-389 (1987)

29. Happel, D.: Triangulated Categories in the Representation Theory of Finite-dimensional Algebras, London Mathematical Society Lecture Note Series, vol. 119. Cambridge University Press, Cambridge (1988) 
30. Happel, D.: Auslander-Reiten triangles in derived categories of finite-dimensional algebras. Proc. Amer. Math. Soc. 112, 641-648 (1991)

31. Hille, L., Perling, M.: Tilting bundles on rational surfaces and quasi-hereditary algebras. Ann. Inst. Fourier (Grenoble) 64, 625-644 (2014)

32. Holm, T.: Derived equivalence classification of algebras of dihedral, semidihedral, and quaternion type. J. Algebra 211, 159-205 (1999)

33. Kalck, M.: Gentle two-cycle algebras and stratifications of derived module categories (2015), preprint

34. Keller, B.: Deriving DG categories. Ann. Sci. École Norm. Sup. (4) 27, 63-102 (1994)

35. Koenig, S., Zimmermann, A.: Derived Equivalences for Group Rings. Lecture Notes in Math., vol. 1685, p. $x+246$. Springer, Berlin (1998). With contributions by B. Keller, M. Linckelmann, J. Rickard and R. Rouquier

36. Orlov, D.: Derived categories of coherent sheaves and triangulated categories of singularities, Algebra, Arithmetic, and Geometry: in Honor of Yu. I. Manin. Vol. II (Y. Tschinkel and Y. Zarhin, eds.), Progress Mathematics, vol. 270, pp. 503-531. Birkhäuser Boston, Inc., Boston, MA (2009)

37. Rickard, J.: Morita theory for derived categories. J. London Math. Soc. (2) 39, 436-456 (1989)

38. Rickard, J.: Derived categories and stable equivalence. J. Pure Appl. Algebra 61, 303-317 (1989)

39. Ringel, C.M.: The repetitive algebra of a gentle algebra. Bol. Soc. Mat. Mexicana (3) 3, 235-253 (1997)

40. Schröer, J., Zimmermann, A.: Stable endomorphism algebras of modules over special biserial algebras. Math. Z. 244, 515-530 (2003)

41. Skowroński, A., Waschbüsch, J.: Representation-finite biserial algebras. J. Reine Angew. Math. 345, 172-181 (1983)

42. Vossieck, D.: The algebras with discrete derived category. J. Algebra 243, 168-176 (2001) 